\title{
Specific Contact Resistance Measurements of Metal-Semiconductor Junctions
}

\author{
N. Stavitski, ${ }^{1}$ M. J. H. van Dal, ${ }^{2}$ R.A.M. Wolters, ${ }^{1,3}$ A.Y. Kovalgin, ${ }^{1}$ J. Schmitz ${ }^{1}$ \\ ${ }^{1}$ MESA+ Institute for Nanotechnology, Chair of Semiconductor Components, \\ University of Twente, Enschede, The Netherlands \\ ${ }^{2}$ Philips Research Leuven, IMEC, Kapeldreef 75, B-3001 Leuven, Belgium \\ ${ }^{3}$ Philips Research, Prof. Holstlaan 4, 5656 AA Eindhoven, The Netherlands
}

\begin{abstract}
Our research comprises the manufacturing of test structures to characterize the metal-semiconductor junctions with a number of techniques and materials. An extensive subsequent physical and electrical testing of the junctions is carried out. We present our first results on specific silicide-to-diffusion contact resistance characterization using the known Scott's Transmission Line Model (TLM) and our approach, considering particular geometry, with NiSi and PtSi as the silicides.
\end{abstract}

\section{INTRODUCTION}

Future improvements of IC technology demand the introduction of many new materials i.e. for metalsemiconductor junctions in particular and studying both the technology and properties of such junctions in a broad sense. Metal-semiconductor junctions are essential to any electronic system containing semiconductors. These junctions have always been a topic of research [1-3].

The performance of MOS circuits depends strongly on transistor drive current. The drive current of the transistor is determined by the total device resistance, which consists of the channel resistance and the parasitic resistances associated with diffusions and contacts. As device dimensions shrink in each new technology generation, contact resistance scales as a power of the reciprocal dimensions [4]. It is expected that the contact resistance between silicide and source/drain region will dominate the total series resistance. This has serious consequences for current drive and device speed.

The silicide must provide low contact resistance to the doped silicon regions. The reduction of this contact resistance and the corresponding specific contact resistance is a large issue in order to not compromise the device performance. Thus the ability to accurately measure the contact resistance is essential to contact process development. For this purpose, a set of test structures was fabricated and specific contact resistance was derived using the Scott model of TLM [5] and a correction for the current distribution.

\section{DESCRIPTION OF THE MEASUREMENT TECHNIQUES}

\section{A. Transmission Line Model}

The theoretical expression of the contact resistance contribution to the series source and drain resistance is expressed as [5]:

$$
\left(R_{\mathrm{c}}\right)_{\text {Transistor }}=R_{\mathrm{cs}}+R_{\mathrm{cd}}=\frac{2 \sqrt{\rho_{\mathrm{c}} R_{\mathrm{s}}}}{W \tanh \left(L / L_{\mathrm{c}}\right)}
$$

where $\rho_{c}$ is the specific contact resistance from the silicide to diffusion. The diffusion layer under the silicide is characterized by $R_{\mathrm{s}}$, the sheet resistance under the silicide, $W$ is the transistor width and $L$ is the length of the silicide contact. $L_{\mathrm{c}}$ is the transfer length defined as $L_{\mathrm{c}}=\sqrt{\rho_{\mathrm{c}} / R_{\mathrm{s}}}$.

The current tends to stay in the silicide as long as possible before moving into the silicon over a distance corresponding to the transfer length $L_{\mathrm{c}}$. Two limiting cases for the contact resistance could be expressed. For $L \gg L_{\mathrm{c}}$, (1) is reduced to:

$$
R_{\mathrm{cs}}+R_{\mathrm{cd}}=\frac{2 \sqrt{\rho_{\mathrm{c}} R_{\mathrm{s}}}}{W}=R_{0}
$$

The limit expressed by (2) corresponds to the ideal case when the contact contribution of the source and drain to the series resistance is independent of the silicided contact length $(L)$. For $L<<L_{\mathrm{c}}$, (1) is reduced to:

$$
R_{\mathrm{cs}}+R_{\mathrm{cd}}=\frac{2 \rho_{\mathrm{c}}}{L W}
$$

The limit (3) denotes the case when the contact resistance depends on the contact area. The extraction of the contact resistance contribution to the series source and drain resistance cannot be done using transistor measurements; appropriate test structures are necessary for contact resistance evaluation. The transmission line structure [5] is a useful and simple method to accurately describe the behavior of the silicide contact resistance.

Silicide to silicon contact resistance is investigated using a set of dedicated test structures with silicided segments of varying lengths based on the Scott model 
of the Transmission Line Model (TLM) [6]. The TLM structure consists of alternating silicided and unsilicided segments formed by using a silicideblocking mask (Fig. 1).

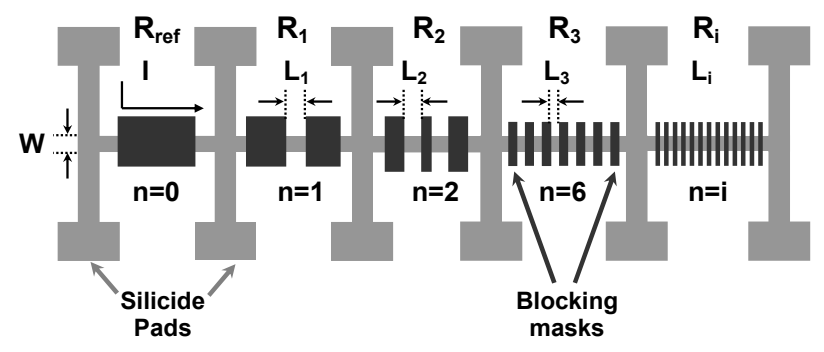

Fig. 1. Example of a TLM layout structure (top view).

The measurement technique involves forcing the current through the reference structure not interrupted by silicide segments in series with the structures interrupted by one, two or $n$ silicided segments and measuring the voltage drop across each structure. As the structures have been designed to have equal silicided and non-silicided segments lengths, the difference between the reference resistance and the other resistances is attributed to the contact resistance contribution. Thus, the contact resistance of each structure measured experimentally is expressed as:

$$
\left(R_{\mathrm{c}}\right)_{\text {meas }} W=\left(\frac{R_{\mathrm{i}}-R_{\mathrm{ref}}}{n}\right) W
$$

where $R_{\mathrm{i}}$ is the resistance of the structure interrupted by $n$ silicided segments, $R_{\text {ref }}$ is the resistance of the reference structure and $W$ is the structure width. The theoretical expression of the silicide-to-silicon contact resistance for the test structure as stated by Scott is given as:

$$
\left(R_{\mathrm{c}}\right)_{\text {meas }}=\frac{2 \sqrt{\rho_{\mathrm{c}} R_{\mathrm{s}}} \tanh \left(L / 2 L_{\mathrm{c}}\right)}{W}
$$

where $\rho_{c}=L_{\mathrm{c}} R_{0} W / 2$ is the specific contact resistance, $R_{\mathrm{s}}$ is the sheet resistance under the silicide, $W$ is the structure width and $L$ is the length of the silicided segment. $L_{\mathrm{c}}$ is the transfer length. Once more, two limiting cases for the contact resistance can be expressed, for $L \gg L_{\mathrm{c}}$ equation (5) reduces to

$$
\left(R_{\mathrm{c}}\right)_{\text {meas }}=\frac{2 \sqrt{\rho_{\mathrm{c}} R_{\mathrm{s}}}}{W}=R_{0}
$$

The limit expressed by (6) corresponds to low contact resistance thus all the current flows through the silicide contact. The value of $R_{0} W$ obtained with the transmission line structure using the long silicided segments equals to limit (2) achieved with a transistor with long silicided contact. For $L<<L_{\mathrm{c}}$ equation (5) reduces to:

$$
\left(R_{\mathrm{c}}\right)_{\text {meas }}=\frac{L}{W} R_{\mathrm{s}}
$$

The limit of (7) shows the case when only a fraction of the current will flow in the silicided segment of the TLM. In the transistor all the current has to enter the silicide, resulting in lower drive current when $L<<L_{\mathrm{c}}$. By plotting $\left(R_{\mathrm{c}}\right)_{\text {meas }} W$ as a function of silicided length $L$, the contact resistance saturates for $L \gg L_{\mathrm{c}}$ to the maximum value $R_{0} W$.

The TLM contact resistance given by the equation (5) can be expressed as:

$$
\frac{R_{0}+\left(R_{\mathrm{c}}\right)_{\text {meas }}}{R_{0}-\left(R_{\mathrm{c}}\right)_{\text {meas }}}=\exp \left(\frac{L}{L_{\mathrm{c}}}\right)
$$

Plotting $\ln \left(\left(R_{0}+R_{\mathrm{c}}\right) /\left(R_{0}-R_{\mathrm{c}}\right)\right)$ as function of $\mathrm{L}$ allows to extract the transfer length $L_{\mathrm{c}}$. Using extracted $L_{\mathrm{c}}$ and $R_{0}$ values, the resistance of the test structure $\left(R_{\mathrm{c}}\right)_{\text {meas }}$ can be plotted to fit the theoretical curve and, therefore, the specific contact resistance $\rho_{c}$ can be calculated.

\section{B. Current direction influence}

The TLM derivation as shown in [6] requires the assumption of the electric current through the interface between the silicide and diffusion areas to be vertical. This corresponds to the first extreme case depicted in Fig. 2a. Although, using the modern method of growing silicide, it is known that the structures are built in into the diffusion area, so the assumption of the current to be vertical is not accurate. Considering the second extreme case, we can assume a horizontal current injection, for a better description of the realized structures with embedded silicide areas (Fig. 2c).

a

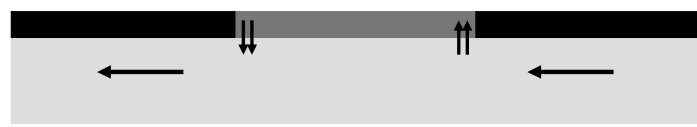

b

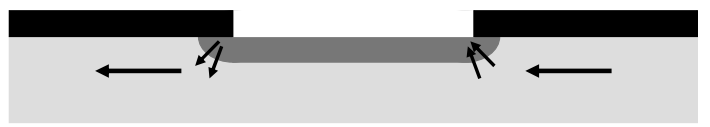

C

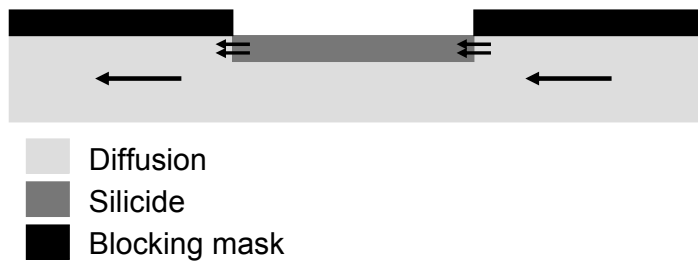

Fig. 2. Current directions for different cases of silicide formation: (a) Scott model; (b) realized structure; (c) first approach to horizontal current distribution.

The realized structure (see the TEM image in Fig 5 and the schematic representation in Fig 2b) can be 
described using a resistor network depicted in Fig. 3. Such a network should especially be applied to the rounded corners of the silicide-to-diffusion transition regions. Using this approach, one can calculate $2 \mathrm{D}$ current distributions in different materials. The number of horizontal sub-layers in such a scheme (i.e. horizontal resistor lines; note that only two sub-layers are shown in Fig 3) to be varied depending on actual dimensions and accuracy requirements.

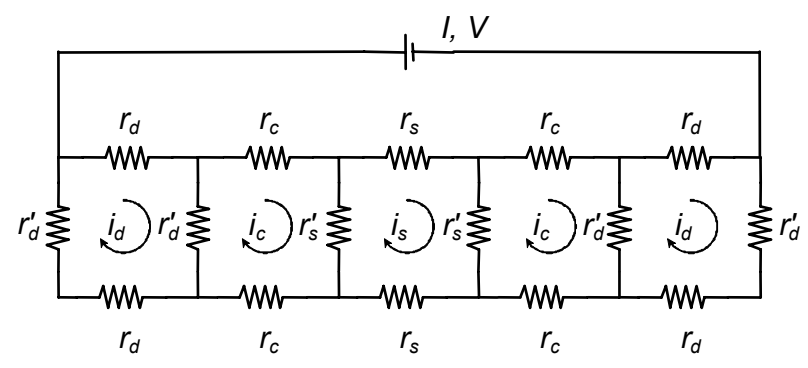

Fig. 3. Schematic representation of the realized structure with one silicide fragment only (Fig. 2b); $r_{\mathrm{d}}$ and $r_{\mathrm{d}}^{\prime}-$ are diffusion resistances, $r_{\mathrm{s}}$ and $r_{\mathrm{s}}^{\prime}-$ silicide resistances, $r_{\mathrm{c}}-$ silicide to diffusion contact resistance and $i_{\mathrm{s}}, i_{\mathrm{c}}, i_{\mathrm{d}}$ are currents for the corresponding loops.

In terms of the proposed loop-current model, using Kirchoff's laws for current and voltage and Ohm's law it can be shown that:

$$
\begin{aligned}
& 2\left(I-i_{\mathrm{d}}\right) r_{\mathrm{d}}+2\left(I-i_{\mathrm{c}}\right) r_{\mathrm{c}}+\left(I-i_{\mathrm{s}}\right) r_{\mathrm{s}}=V \\
& 2 i_{\mathrm{d}}\left(r_{\mathrm{d}}+r_{\mathrm{d}}^{\prime}\right)-I r_{\mathrm{d}}-i_{\mathrm{c}} r_{\mathrm{d}}^{\prime}=0 \\
& \left(2 r_{\mathrm{c}}+r_{\mathrm{d}}^{\prime}+r_{\mathrm{s}}^{\prime}\right) i_{\mathrm{c}}-I r_{\mathrm{c}}-r_{\mathrm{d}}^{\prime} i_{\mathrm{d}}-r_{\mathrm{s}}^{\prime} i_{\mathrm{s}}=0 \\
& 2 i_{\mathrm{s}}\left(r_{\mathrm{s}}+r_{\mathrm{s}}^{\prime}\right)-I r_{\mathrm{s}}-2 i_{\mathrm{c}} r_{\mathrm{s}}^{\prime}=0 .
\end{aligned}
$$

The loop currents do not change for the same regions (e.g. for all diffusion regions) due to the symmetry of the structure. This approach based on a variable resistor network will be developed in our future work. In this paper we will only consider the simplest case shown in Fig 2c.

The input current $I$ and voltage drop across the structure $V$ can be measured using $R_{\text {ref }}$ and $R_{1}$ from the TLM layout, which eliminates the diffusion resistance $\left(r_{d}\right.$ and $\left.r_{\mathrm{d}}^{\prime}\right)$ contribution:

$$
V / I=R=R_{1}-R_{\mathrm{ref}}
$$

As the resistance of the diffusion area is eliminated, the resistor network can be simplified to the network depicted in Fig 4. Here we assume that the horizontal current distribution is uniform in the vertical direction inside the silicide. The current can only flow inside the silicide and not in the diffusion area under the silicide.

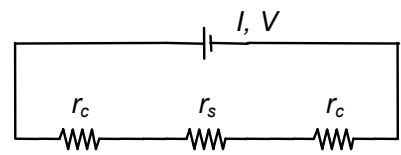

Fig. 4. Schematic representation of one-silicide fragment shown in Fig. 2c.

The silicide resistance $r_{\mathrm{s}}$ can be calculated from the measured silicide sheet resistance $r_{\mathrm{s} \text { (sheet) }}$ on the same wafer using one of the sheet resistance measurement techniques and silicide geometry factors - the length $(L)$ and the width $(W)$ :

$$
r_{\mathrm{s}}=r_{\mathrm{s}(\text { sheet })} L / W
$$

The contact resistance $r_{c}$ can then be calculated from Ohm's law:

$$
I\left(2 r_{\mathrm{c}}+r_{\mathrm{s}}\right)=V
$$

\section{EXPERIMENTAL RESULTS}

For the contact resistance study (100) p-type Si wafers were used as starting material on which active areas were defined by Shallow Trench Isolation. Channels were defined by I-line lithography and doping concentrations were achieved by low-dose well implantations B (180 keV) and P (380 keV) for the Pwell and N-well respectively. Highly Doped Drain (HDD) implantations were carried out for corresponding wells: As $(20 \mathrm{keV})$ for NHDD and B ( $2 \mathrm{keV}$ ) for PHDD. For the TLM, a silicide blocking layer $\left(\mathrm{SiO}_{2} / \mathrm{Si}_{3} \mathrm{~N}_{4}\right)$ was deposited and patterned using I-line lithography. The segment lengths range from 0.05 to $3 \mu \mathrm{m}$ and have a width of $8 \mu \mathrm{m}$. The smaller segments $(0.25 \mu \mathrm{m}$ to $50 \mathrm{~nm})$ were defined with ebeam lithography. Each of the four structures has $n$ silicided segments, where $n$ equals $1,2,6,12,15,20$, 30, 40 and 60 (Fig. 1). Finally, a 10-nm thick Ni layer or a $13-\mathrm{nm} \mathrm{Pt}$ was deposited and silicide was formed by either a two-step anneal $\left(300{ }^{\circ} \mathrm{C}\right.$ for $30 \mathrm{~s}+470{ }^{\circ} \mathrm{C}$ for $30 \mathrm{~s})$ for $\mathrm{NiSi}$ or a one-step anneal $\left(500{ }^{\circ} \mathrm{C}\right.$ for 30 sec) for PtSi. In both cases the unreacted metal was selectively removed by wet etching.

Silicide profiles and lengths for the smaller segments were verified by TEM-analysis (see e.g. Fig. 5). The samples were prepared by a combination of mechanical polishing and FIB200 technique (Focused Ion Beam), depositing a layer of low-temperature PECVD silicon nitride prior to preparation as a protection layer and a thin Pt layer to avoid charging. In the FIB equipment a $1.5 \mu \mathrm{m}$ thick Pt layer is deposited on each structure to protect the sample during FIB milling. The TEM-analysis was performed on a FEI Tecnai F30ST TEM operated at $300 \mathrm{kV}$. The silicide profiles are in agreement with structure (b) in Fig. 2. The actual segment lengths are always larger 
than the defined ones due to lateral expansion of silicide, ranging from 10 to $20 \mathrm{~nm}$ on each side.

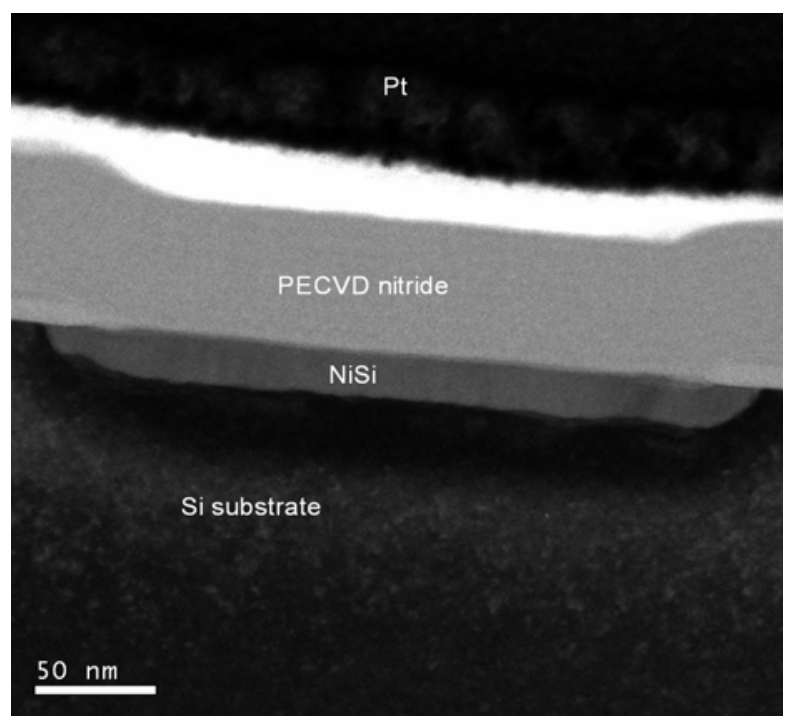

Fig. 5. Example of a X-section TEM image of NiSi profile for $n=12$, showing the lateral silicide formation.

According to the Scott model the transfer length $\left(L_{\mathrm{c}}\right)$ value was extracted using expression (8) and the actual silicide lengths derived from TEM (Fig. 6).

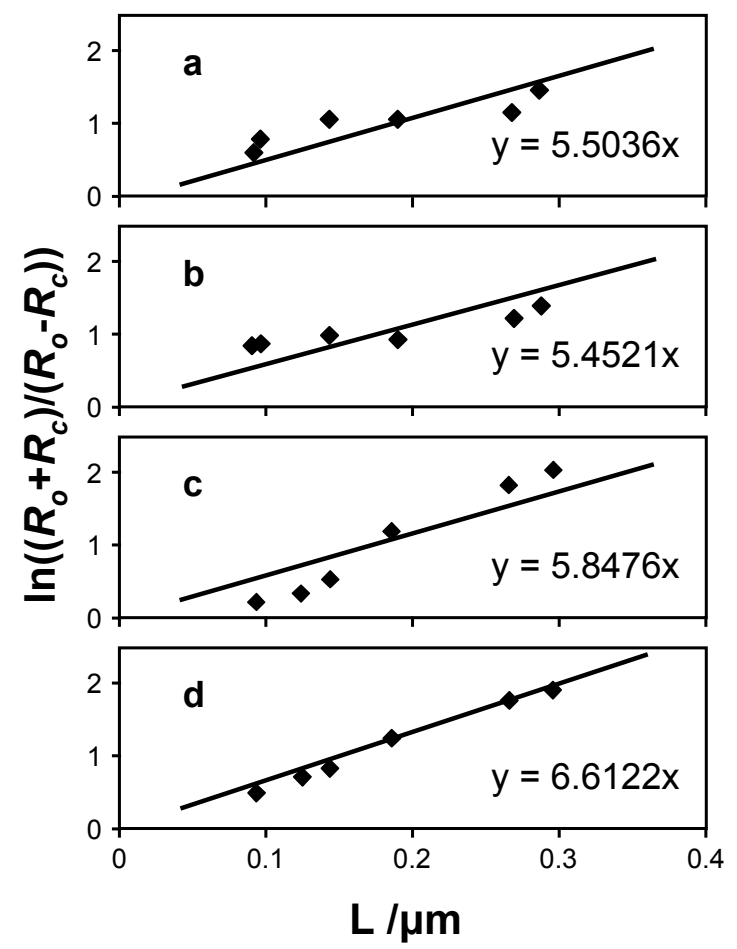

Fig. 6. $\ln \left(\left(R_{0}+R_{\mathrm{c}}\right) /\left(R_{0}-R_{\mathrm{c}}\right)\right)$ as a function of silicided segment length. The slope equals to $1 / \mathrm{L}_{\mathrm{c}}$ reveals $\mathrm{L}_{\mathrm{c}}$ extraction for (a) NiSi/ntype silicon (b) NiSi/p-type silicon (c) PtSi/n-type silicon (d) PtSi/ptype silicon

Using extracted $L_{\mathrm{c}}$ and $R_{0}$ values, the specific contact resistance for NiSi is calculated to be $1.8 \times 10^{-8} \Omega \cdot \mathrm{cm}^{2}$ (to n-type silicon) and $3.9 \times 10^{-8} \Omega \cdot \mathrm{cm}^{2}$ (to p-type silicon) and for PtSi is $5.3 \times 10^{-8} \Omega \cdot \mathrm{cm}^{2}$ (to n-type silicon) and $9.3 \times 10^{-8} \Omega \cdot \mathrm{cm}^{2}$ (to p-type silicon).
The fit of experimental data for NiSi and PtSi with the Scott model for $R_{\mathrm{c}}(5)$ when the values extracted for $L_{\mathrm{c}}$ and $R_{0}$ are implemented may be found on Fig. 7-8 respectively.

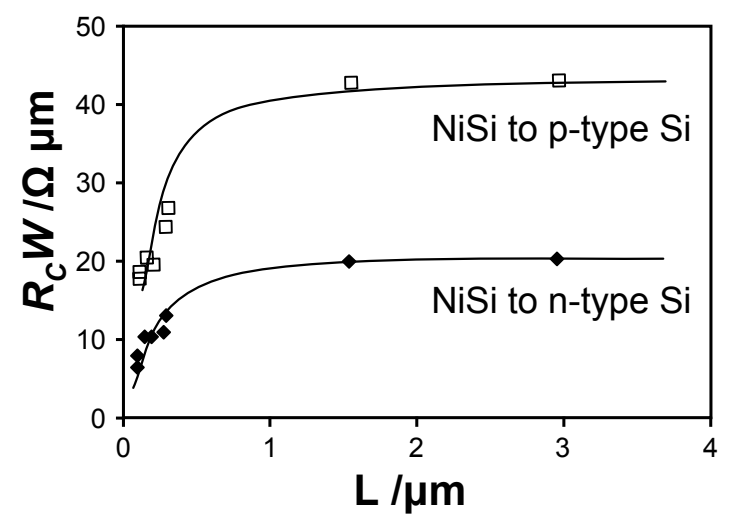

Fig. 7. Contact resistance values for $\mathrm{NiSi}$, measured (dots) vs. calculated (lines) using the Scott model

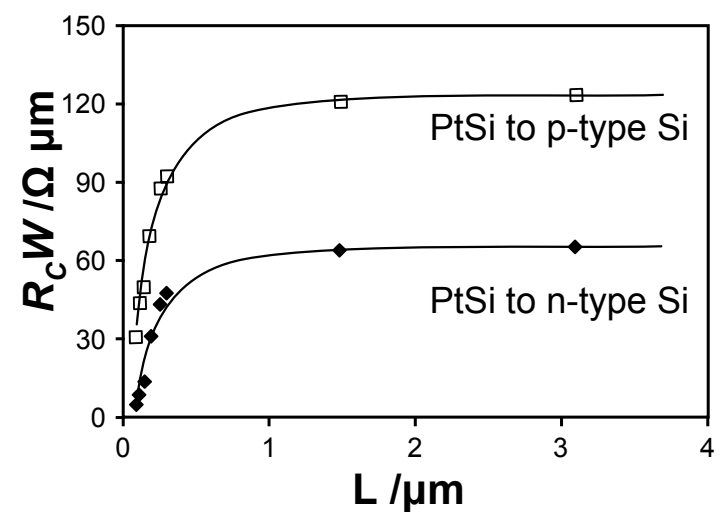

Fig. 8. Comparison of the curve fit values with the measured values for PtSi

The values of $r_{\mathrm{s} \text { (sheet) }}$ were measured using Van der Pauw structures. From the equations (10)-(12), values of $r_{\mathrm{c}}$ were extracted. The specific contact resistance can be calculated as a product of $r_{\mathrm{c}}$ and the interface contact area between the silicide and diffusion regions. The specific contact resistance was found to be $0.20 \times 10^{-8} \Omega \cdot \mathrm{cm}^{2}$ for NiSi (to p-type silicon) and $0.59 \times 10^{-8} \Omega \cdot \mathrm{cm}^{2}$ for PtSi (to p-type silicon). The values for the n-type case are lower compared to the $\mathrm{p}$ type silicon. However, the dimensions $L$ and $W$ were not known with sufficient precision to compute a reliable value of specific contact resistance on n-type silicon.

\section{DISCUSSION \& CONCLUSIONS}

The contact resistance between $\mathrm{NiSi}$ and $\mathrm{PtSi}$ and highly doped silicon regions ( $\mathrm{N}$ and $\mathrm{P}$ ) was studied making use of the TLM test structure. The results were analyzed using two methods - the Scott Model (assuming vertical current flow through the diffusion/silicide interface) and the approach modified for the case of a horizontal current flow through the 
interface. Specific contact resistances obtained by the two methods are different. This significant difference is explained by different assumptions for the geometrical factors of the structures and consequently different current distributions. The Scott Model assumes vertical current distribution while our approach ultimately assumes a horizontal distribution. According to TEM analyses the real structure and therefore the current distribution is more complicated. Here we present only the two boundary cases (as depicted in Fig. 2). Future research will focus on a model with a 2D current distribution for the realized structures. The results will be compared also with different measurement techniques like Kelvin CrossBridge Resistor (KCBR) [7, 8].

\section{ACKNOWLEDGEMENTS}

This project is supported by Philips Research Leuven. Monja Kaiser is acknowledged for the TEM work.

\section{REFERENCES}

[1] C. Wang, J.P. Snyder, and J.R. Tucker, Appl. Phys. Letters, vol. 74, no.8, pp.1174-1176, 1999.

[2] J. Kedzierski, M.Ieong, X. Peiqi, J. Bokor, T.J. King, and C. Hu, in 2001 IEEE Int. SOI Conf. Proc., pp. 21-22, 2001

[3] A.Y. C. Yu, Solid-stat. Electron, vol.13, pp. 239247, 1970.

[4] C.M. Osburn, K.R. Bellur, Thin Solid Films, vol.332, pp. 428-436, 1998

[5] D.B. Scott, R. A. Chapman, C. Wei, S. S. MahantShetti, R.A. Haken and T.C. Holloway, IEEE Trans. on Electron Devices, pp. 562-574, 1987

[6] D.B. Scott, W.R. Hunter, and H. Shichijo, IEEE Trans. Electron Devices, vol. Ed-29, pp.651-661, 1982.

[7] W. M. Loh, K. Saraswat, and R.W. Dutton, IEEE Electron Device Lett., vol. EDL-6, no.3, pp. 105108, 1985.

[8] W. M. Loh, S.E. Swirhun, E. Crabbe, K. Saraswat, and R.M. Swanson, IEEE Electron Device Lett., vol. EDL-6, no.3, pp. 105-108, 1985 